\title{
EL PAPEL DE LAS EMOCIONES PARA ENTENDER LA CENTRALIDAD DE ELECTRA EN LA TRAGEDIA DE SÓFOCLES
}

\author{
Benjamín Franzani \\ Universidad de los Andes. Chile
}

Resumen: Centrar el estudio de la Electra de Sófocles en las emociones que caracterizan a la protagonista permiten dar un sentido de unidad a la obra y entender las relaciones que a propósito de dichas emociones se traban entre los personajes, lo que enfatiza el papel central que Electra despliega en la obra, hasta el punto de que el interés de la obra no está tanto en la trama, como en el ethos de su protagonista

Palabras clave: comunidad emocional - emociones - Electra - odio - ira - lamento - juicios - cognitivismo - recepción - oralidad.

\section{THE ROLE OF EMOTIONS TO UNDERSTAND THE CENTRALITY OF ELECTRA IN SOPHOCLES' TRAGEDY}

Abstract: To center the study of Sophocles' Electra in the emotion which characterizes the protagonist allows us to give a sense of unity to the play and to understand the relationships which occur between the characters in behalf of this emotions, which stress the central role that Electra displays in the play, until the point that interest is not so much in the plot as in the ethos of its protagonist.

Keywords: emotional community - emotions - Electra - hatred - anger - grief judgment - cognitivism - reception - orality.

Recibido: 24.10.2019 - Aceptado: 30.04.2020

Correspondencia: Benjamín Franzani García

Email: Correo: bfranzani@uandes.cl

Universidad de los Andes - Chile

ID Orcid: https://orcid.org/0000-0002-4819-986X 
T a Electra de Sófocles es una tragedia que frecuentemente ha resultado difícil de interpretar para los críticos. Para abordarla, nos acercaremos a ella tratando de ponernos en el lugar de los oyentes: como toda tragedia, Electra fue compuesta en primer lugar para ser representada, para ser vista y oída. Por ende, es lógico suponer que Sófocles, si pensaba transmitir alguna idea con ella, tuviese en mente esta situación de oralidad.

La oralidad de su recepción hace que para entender la tragedia se vuelvan especialmente importantes las imágenes y las emociones, que son los vehículos privilegiados para llegar a un público, que solo oirá y verá una vez cada escena. Ellas van dejando en la memoria una sensación general sobre la acción y sus protagonistas que ayudan a interpretar la obra misma. Como afirma Helen Foley "no audience can depart without remembering the lamentations to which Orestes tries to close his ears, nor the chorus' open admiration for Electra and its celebration of her choice" (2001, p. 168).

En una obra como Electra el papel de las emociones es evidente, pues los lamentos y diálogos apasionados la cruzan por completo. Sin embargo, considerar la emocionalidad de la obra simplemente como algo superficial, como un elemento complementario o un adorno sofisticado para mantener la atención del público sobre la acción sería un error.

Las emociones, especialmente en el mundo de la oralidad, están lejos de ser simple irracionalidad. Al contrario, a través de ellas es posible identificar qué es lo que una persona o una sociedad consideran bueno y valioso, y qué comportamientos, en cambio, son reprensibles: "emotions are assessments of weal or woe, and thus tell not only to ways of communicating but also to the whole set of goals that and individual, a group, a community sets for itself and considers to be in its interest'(Rosenwein, 2003, p. 8).

Esta idea resulta ser muy cercana a la que los mismos griegos tenían respecto de las emociones, para quienes, más que impulsos externos, eran herramientas persuasivas $\mathrm{y}$, sobre todo, apreciativas:

The emotions, as opposed to drives and appetites, depend on the capacity for symbolization. For the Greeks, persuasion was central to the idea of an emotion, whether in the law courts, in political assemblies, or in the various therapies that relied on verbal interactions to change the judgments that are constitutive o passions (el énfasis es nuestro) (Konstan, 2001, p. XII). 
Sófocles era consciente del papel que juegan las emociones, como se puede colegir de los discursos que intercambian los personajes en la tragedia, en los que con frecuencia se alude a los sentimientos que los argumentos producen. Al examinar las emociones y lo que quiso transmitir a su público a través de ellas, hay que considerar que él y sus oyentes pertenecían a lo que Bárbara Rosenwein denomina una misma "comunidad emocional": reconocen unas mismas normas de conducta emocional y por ende unas mismas metas, valores e intereses que les unen como grupo (Rosenwein, 2006, p. 24).

La primera aproximación, pues, tendrá que ser hacia el contexto de la comunidad que presenciaba el teatro, para intentar ver la obra como ellos la entendían. Luego, podremos centrarnos en la emocionalidad misma.

Analizar la obra desde este punto de vista nos debiera orientar hacia aquello que es esencial en ella, que no es otra cosa que la figura misma de Electra, más aun, incluso, que la misma acción dramática, según veremos.

\section{Las emociones en su contexto}

Adelantábamos que para los antiguos griegos las emociones tenían un elemento apreciativo. Así lo entiende Konstan: “evaluation presupposes values, and insofar as emotions are a function of value judgments, they will vary from one individual to another and according to the collective values of particular communities" (Konstan, 2001, p. 24). El juicio no es la emoción misma, pero la precede (2001, p. 21), así como la emoción puede arraigar aún más el juicio (2001, p. 37), el que a su vez dependerá en buena medida del contexto en que se encuentra (2001, p. 30). "Given -concluye Konstan - that judgment and belief are central to the dynamics of the emotions as Aristotle conceives them, it is natural that an understanding of the pathe should form part of the art of persuasion" (2001, p. 27).

Prueba, en parte, de que esta concepción cognitiva de las emociones no era solo idea del Filósofo, es el famoso Encomio de Helena en el que Gorgias defiende la fuerza persuasiva de las emociones: "la palabra es un poderoso soberano que con un cuerpo pequeñísimo y del todo invisible lleva a término las obras más divinas. Pues es capaz de hacer cesar el miedo y mitigar el dolor, producir alegría y aumentar la compasión" (Gorgias, 2011, párr. 8). La "palabra" aquí, esto es el logos o razón, tiene para Gorgias clara influencia no solo sobre la racionalidad sino también sobre los sentimientos de los oyentes, siendo capaz de suscitarlos y guiarlos según las razones que quien maneja la palabra va afirmando. 
Cuando oigamos a los personajes hablar en Electra no debemos perder de vista estas consideraciones: por un lado, las emociones tienen un carácter social para los griegos, pues son consideradas siempre en relación a los otros y especialmente al juicio sobre la posición social en que se está respecto del interlocutor; y por otro lado, eran utilizadas como herramientas de persuasión: se esperaba no solo que ante un argumento se asintiera con la razón, sino también que se lo sintiera.

Las emociones se relacionan, pues, con un juicio, y no con cualquiera, sino con una cierta apreciación, basada en valores previos, del contexto que se presenta a la persona, dirigiendo la respuesta del individuo -0 de la sociedad - hacia las que considera sus metas, a la afirmación de sus principios.

En el caso griego, y particularmente en el de la tragedia que nos ocupa, un principio fundamental de la ética griega es el que Mary Whitlock sintetiza en "ayuda a tus amigos y has daño a tus enemigos", estrechamente relacionado con una concepción de justicia como represalia. "In particular - continúa Whitlock - both Help Friends/Harm Enemies and retaliatory justice are rooted in passion ${ }^{1} "(1989$, p. 1).

Estos principios que mueven los actos de los personajes en escena son comunes tanto a la época arcaica como a la clásica, sin embargo, la manera en que son vividos y apreciados es necesariamente distinta en uno u otro periodo, aunque la matriz cultural siga siendo la misma.

Afirma al respecto John Gould que

The development in new forms of social organization and in new political institutions that marks the transition from 'archaic' world of the seventh and sixth centuries to the 'new' societies of fifth century Greece ... brought about a shift of consciousness, a new spirit of rational and critical enquiry, a new readiness, even a compelling need, to re-examine and to attack traditional assumptions that brought the societies of ancient Greece ... The experience of open discussion

1 Esto implicaría, según esta autora, un cuestionamiento de la racionalidad de las decisiones morales y de la relación entre justificación moral y emociones, con lo que no estamos del todo de acuerdo. El hecho de que los principios de la ética griega estén enraizados en la emoción habla más bien de la acertada visión del cognitivismo que mira a las emociones no como impulsos irracionales, sino como parte de la valoración de un juicio que encamina a conseguir una meta o afirmar, precisamente, un principio. 
of fundamental assumptions about society and about political and legal decision-making had created in men a radical and cumulative scepticism about traditional wisdom and a new sophistication in the analysis of human behaviour and motivation. Added to this, it is argued, comes a increasing awareness of ... a consuming interest in the varieties of human experience and of human solutions of the problem of experience (Gould, 1983, p. 34).

Sófocles se enmarca en esta corriente, tratando temas tradicionales, en los que sus personajes se mueven en un ambiente arcaico, al que sin embargo por eso mismo cuestiona, interpreta, analiza. Como el mismo Gould dirá más adelante, los trágicos no se desmarcan totalmente de la tradición homérica, y no pueden ser entendidos sin esta, el "momento trágico" del siglo $\mathrm{V}$ es un momento de ambivalencia (40 y ss.).

En su obra, quizá lo que más resulta evidente en Sófocles es su interés en los personajes y en sus dilemas morales. El héroe en Sófocles ha sido objeto de profusa investigación. De hecho, cada una de las obras que nos han llegado de Sófocles está dominada por una o unas figuras que pretenden encarnar un patrón de creencias heroicas pese a la oposición externa que enfrentan (Goldhill, 1986, p. 154). Este es también el caso de su Electra.

El héroe de Sófocles destaca por su intransigencia y extremismo al llevar hasta las últimas consecuencias las ideas de justicia que enarbola. Esto lo hace particularmente dramático, pues precisamente en una sociedad que Konstan describe como increíblemente competitiva (2001, p. 259) el protagonista es perfectamente consciente de su posición social, de que todos mirarán su decisión y está dispuesto en llevar hasta las últimas consecuencias, sin cómodas morigeraciones de ninguna clase, sin buscar excepciones, la regla social que fija su dignidad y que da pie a su trágica intransigencia. ¿Servían, quizá, los personajes representados en escena para discutir a lo que podrían llegar las propias presuposiciones del público, llevadas al extremo? Es una posibilidad, nada despreciable si tomamos en cuenta las mutuas relaciones entre épica y tragedia, y el carácter ejemplar que juega en la épica la figura del héroe. En efecto:

Yet the idea that the poet teaches through the example of his characters also presupposes that these characters may be evaluated as models of behavior, and hence may legitimately be subjected to moral scrutiny. The plays themselves invite us to locate their characters 
within such an evaluative framework, not only through the kind of action that they represent, but through the characters' frequent deployment of moral language and argument (Whitlock Blundell, 1989, p. 15).

Las emociones de los héroes de Sófocles vendrían de ese modo a confirmar y a reforzar esta apreciación de extremismo, planteando el problema ético como el centro de la tragedia. El contexto extremo en que se encuentran los protagonistas los lleva a juicios y actitudes, secundadas por emociones, igualmente extremos, pero que sin embargo no son vistos como algo ajeno para los espectadores, capaces así de empatizar con ellos al compartir unas mismas valoraciones basales sobre la sociedad, y de emitir un juicio sobre lo que se les presentaba en la ficción.

\section{El objeto de la obra: ¿la acción o el personaje?}

Si observamos la acción dramática de la tragedia, podríamos quizá concluir que el papel de Electra es totalmente superfluo: ninguna de sus acciones tiene consecuencia alguna sobre el desenlace de la trama. Ésta se hubiese desarrollado del mismo modo, y quizá hasta con mayor rapidez, sin la presencia de la hermana mayor de Orestes. Sin embargo, la tragedia lleva su nombre y gran parte de la acción se centra en ella y en sus diálogos, lo que hace preguntarnos si lo central en esta obra es la trama, la acción dramática, o más bien su personaje principal. Parecida pregunta se hace Foley al tratar esta obra (2001, p. 146), respondiendo a lo largo de su trabajo que el foco de la obra está en representar en escena una ética de la vendetta femenina, en representar la búsqueda de justicia por parte de una mujer a través del lamento (2001, pp. 150, 170). Es decir, concluimos nosotros, el motivo de la obra no está en la acción dramática llevada a cabo por Orestes (la venganza) sino en el personaje mismo de Electra y lo que ella hace, lamentarse, aunque no tenga un efecto inmediato sobre la acción.

Esto significa que la trama misma está en función de Electra. Lo que en ella ocurre sirve sobre todo para perfilar cada vez más su figura, para caracterizarla frente al espectador, que finalmente juzgará de acuerdo con las emociones que tal personaje suscite: podemos imaginar a Electra como una imagen viviente de un discurso retórico, que por su pasionalidad extrema nos convence sobre la justicia de su argumento, tal y como Gorgias describía el poder persuasivo de las palabras.

Afirmar esto no implica necesariamente oponerse a la idea aristo- 
télica de que la trama está por sobre los personajes, sino que más bien implica entender mejor las relaciones entre la trama y el ethos. Es posible concebir una trama que tenga por finalidad no una acción, sino resaltar una determinada figura:

Sophoclean stage figures may be treated as bearers of a broadly consistent Aristotelian ethos, or moral and intellectual character. Plot is fundamental for Aristotle, because he sees it as logically prior to the other qualitative parts of tragedy (50a20-5). But he is more interested in dramatic ethos than is sometimes allowed. It is the only serious contender with plot for primacy, remains an easy second of the six 'parts' of tragedy (50a39), receives detailed treatment in its own right, and is inextricably involved in the discussion of plot and action. 'They do not act in order to represent their characters, but they include the characters for the sake of their actions' (50a20-2). Thus despite the logical priority of action over ethos, the two are intimately related. Not only does ethos help to generate action, but action also reveals ethos (Whitlock Blundell, 1989, pp. 16-17).

Considerar de este modo las cosas quita relevancia al punto de que las acciones de Electra no tengan influjo en el final de la acción dramática: los discursos y lamentos de Electra sirven al objetivo de la trama, que es realzar su propio personaje. Y, por lo demás, puede que a nosotros nos parezca que la protagonista no hace nada relevante, pero esto es desconocer la relevancia y función que, de acuerdo con Foley, tenía el lamento de las mujeres en las sociedades en que estaba legitimado el uso de la vendetta. En efecto, el papel de la mujer a la que habían injuriado un pariente cercano era precisamente el de lamentarse, compeliendo con sus llantos a la piedad social para traer sobre el culpable la venganza:

Lamentation has a particular function to play in the jural system of cultures that practice feuds or vendetta justice; it aims to provoke revenge through the awakening of shared pain, through the blurring of boundaries between past and present injustice, between the living and the dead. In a fashion traditional to the function of lamentation both in ancient and modern Greek culture, Electra keeps alive the cause of the dead Agamemnon, awakens the citizens' longing for the return of Orestes, and makes them witnesses to her own view of the past. The women of the chorus are significantly called politides, female citizens (1227); when Electra stirs them to lament, she 
violates contemporary Athenian strictures against women lamenting with nonkin (Foley, 2001, p. 151).

Tomando en cuenta este contexto, que probablemente perviviría aún en la memoria colectiva de los primeros oyentes de Electra, la protagonista deja de ser una figura inútil de la trama, pasando a ocupar un papel central en ella. Pues, podemos decirlo así, la trama trata sobre ella misma, sobre su situación, sobre sus desgracias, sobre la injusticia que busca reparación, venganza.

Por otro lado, el contexto no es únicamente el de la vendetta. Hay todavía algo más, y es el de la transgresión de las normas del luto. Richard Seaford nos llama la atención sobre ello, afirmando que el lamento sin fin de Electra, que se extiende mucho más allá de lo permitido, se debe a una subversión de los límites por ambos "bandos" en conflicto, que la relegan a ella a permanecer permanentemente en "el lugar de los muertos" (Seaford, 1985, p. 317), pasando el lamento mismo a ser un arma en manos de la protagonista:

It is [the lamentation] an instrument in the apparently endless conflict with her mother and stepfather, a response, certainly, to the disruption caused by death, but also an agent of disruption in the family. It is true that she attributes to her lamentation the orthodox purpose of honouring the dead; but in this case the honour derives from the $\lambda v i \pi \eta$ caused to other members of the family. So far from being a response by the kinship group to the disruption caused by a death, a response which though relatively unrestrained is nevertheless contained within an articulated framework of separation and reintegration, the ritual of mourning has been perverted by both sides into a weapon in a conflict within the kinship group, a conflict which is uncontained by any temporal, moral, or ritual limit, and which is intensified by the perversion of the natural relationship between mother and daughter. The blood which Elektra drinks from the soul of her mother is 〈unmixed (786). So far from containing disorder, the ritual is employed in its service (Seaford, 1985, p. 320).

Ambas visiones de la obra son compatibles y realzan un aspecto distinto del mismo elemento central: Electra. Efectivamente, más que teorizar sobre la justicia o injusticia, valga la redundancia, de la justicia por propia mano, Sófocles parece querer desplegar ante nuestros ojos una heroína que 
cumple cabalmente con su papel de mujer que se lamenta, haciendo patente a ojos de toda la polis (y por eso es también tan odiosa a los tiranos: vv. 520-524) el desorden introducido por la impía acción de asesinar al marido y rey. Sin embargo, no se trata tan solo de un lamento fúnebre (como veremos, la emoción que Electra transparenta no es solo "grief", planto por el que se ha ido y no volverá), sino que la situación la ha puesto a ella misma en posición de sufrir una injusta dominación: no solo se trata de un luto sin fin, sino que, sobre todo, de una necesidad de volver las cosas a su justo sitio, trayendo para ello la venganza justiciera o, cuando ésta parezca faltar, buscando propinarla por la propia mano, como nos recuerda Foley que para una mujer era lícito actuar, en ausencia de un vengador de la sangre en la familia (2001, p. 161).

Llegados a este punto, aunando la visión de Seaford sobre centralidad de la destrucción de los límites del lamento fúnebre con la importancia que la vendetta femenina tiene en esta obra según Foley, podemos decir con esta última que "revenge and funerary lamentation are intimately related in tragedy, and women play a public and dominant role in awakening it" (Foley, 2001, p. 26).

\section{El centro: Electra y la emocionalidad}

La posición de Electra en la obra es dramática desde el inicio, manifestándose en un torbellino de pasiones violentas que desbordan y definen a la heroína. Electra, nos dice Foley,

is quite aware of the ambivalent moral position in which she finds herself. If she does not lament and the murderers do not pay the penalty for their crime, she will betray aidōs (shame or reverence) and eusebeia (piety) and her noble birth (245-50, 257; see the choral confirmation of Electra's eusebeia at 464). Yet her piety toward her father has required perverted behavior toward her mother. Electra is ashamed (aischunomai, 254; see also 606-7, 616) that she has been forced $(256,620)$ to do such terrible things (deina, 221, 223; kaka, 308-9, aischra, 621; aikōs, 216); she knows that her disposition is extreme (222), that her behavior could be interpreted as mad (aluein, 135 ; for $a t \overline{\mathrm{e}}$, see $215,224,235$ ), and that she betrays with her aggressive behavior the quintessential female virtue of sōphrosune (selfcontrol, chastity, discretion). In such circumstances it is not possible to be self-controlled or pious (sōphronein or eusebein, 307-8; see also the ironic phronein kakōs, 345); piety toward a mother who 
is an unjust murderess and unmaternal to her children cannot take precedence over piety to her father. From a spatial point of view, the action increasingly forces Electra to come and stay outside-where women and, above all, unmarried virgins do not belong - to pursue her cause (see 516). In contrast to Orestes, who aims with a precise plan at the kairos, for Electra timely wisdom (phronounti kairia, 228 ) is hard to formulate. From her mother's perspective, Electra has shamed her philoi (close relatives, 518) and committed hubris (violence) in speech $(522,613)$; she drinks her mother's life blood like a Fury (785-86) (Foley, 2001, p. 150).

Socialmente, nos diría Seaford, corresponde a Electra este estar "fuera" y separada de los demás, por su condición de "lamentante" (Seaford, 1985, p. 315), sin embargo, las acciones de su madre la han llevado a la situación anómala de un luto que no tiene fin (Seaford, 1985, p. 317) y sus injusticias la han transformado, a Clitemestra, en una enemiga para su propia casa. De este modo, no es solo el lamento fúnebre, con toda su carga de piedad, pena y congoja que implica, lo que define emocionalmente a Electra, sino que a ello hay que agregar también un acendrado odio que está éticamente obligada a sentir contra sus ofensores, según la rigidez de la fórmula que nos recuerda Whitlock (1989, pp. 28-29), odio que, sin embargo, se dirige contra su propia madre, monstruosidad de la que es plenamente consciente y de la que se siente avergonzada (v. 617).

Si juzgáramos a Electra sólo como una hija que se lamenta por la muerte injusta del padre, no la acabaríamos de entender. Por supuesto que esa dimensión está presente, pero no es ni de cerca la más importante para definir a Electra. El lamento de la protagonista, aun tomando pie siempre en la injusticia inicial, el asesinato, se explaya y se refuerza con la descripción de las propias desgracias y la situación deplorable de la Casa de los Atridas. La opresión de una dominación injusta que ha introducido un desorden social intolerable y la ha relegado a ella a una posición que no le corresponde es el centro del lamento de Electra, a quien se la describe en varias ocasiones como colérica (véanse, p.ej. vv. 369-372, 610-611, 792): cólera de la que se deja llevar también al momento de su discusión con Clitemestra, que comienza fría y racional para acabar cediendo a su ira (vv. 605-609).

La lamentación de Electra no es solo llanto funerario: es un instrumento de su venganza, proporcionado a la posición de la mujer en la 
sociedad griega. Como tal, apunta a provocar el enojo y el odio, de los cuales está completamente imbuida Electra misma: "lamentation actively seeks justice through pain and creation of shared values and memories; it aims quite consciously to arouse anger, even hatred" (Foley, 2001, p. 160).

Aunque en ella se den mezcladas más de una emoción, cabe de todos modos preguntarse cuál es el sentimiento dominante en Electra, si lo que siente principalmente es simple enojo o propiamente odio, además de su pena. Pero antes de hacerlo debemos tener en cuenta una peculiaridad del mundo griego: en efecto, podríamos tender a valorar negativamente al personaje al caracterizarlo con emociones que para nosotros son tan negativas como el odio. Sin embargo, no era así en el mundo griego y, por supuesto, no era así para Sófocles y su auditorio. El odio, como pasión, era para los griegos negativo o positivo dependiendo de su ordenación social, aun cuando su destinatario fuese una persona ${ }^{2}$. Así como entre nosotros la ira o enojo puede estar causada y encausada justamente, como en el soldado que defiende a su patria, también en el mundo griego ocurría idéntica cosa con una pasión como el odio. No hemos de olvidar que ésta se enmarca también en el aforismo "ayuda a tus amigos y daña a tus enemigos" que dirigía la ética antigua, y que por ende para el público un héroe o un personaje dramático podía lucir positivamente si su odio era justamente orientado hacia enemigos que por otro lado hubiesen introducido el desorden que el personaje está llamado corregir (cfr. Whitlock Blundell, 1989, pp. 26-31).

Lo primero que oímos de Electra en la tragedia es un sentido lamento:

¡Oh luz inocente y aire que recubres por igual la tierra! Muchas veces escuchaste cantos de duelo y muchas percibiste golpes en el pecho que me hacían brotar sangre, cuando la sombría noche terminaba. Los odiosos lechos de esta casa desdichada son ya conocedores de lo que ocurre durante la noche: cuántas veces gimo por mi infortunado padre, a quien el sangriento Ares no recibió como huésped en tierra

2 En esto diferimos con los antiguos griegos, pues si en una parte importante de la tradición ética occidental actual el odio es visto también como una pasión neutra, cuya valoración última depende de hacia qué está ordenado, sin embargo la opinión cuasi unánime es que el odio puede estar justamente dirigido contra una situación u objeto, por ejemplo al odiar la injusticia, pero jamás es lícito odiar a otra persona aunque se aborrezcan sus actos, precisamente por la intrínseca dignidad de la persona humana. 
extranjera, sino que mi madre y el que comparte su lecho, Egisto, como leñadores a un árbol, le abrieron la cabeza con asesina hacha (vv. 88-99).

Estas palabras iniciales conducen naturalmente a pensar que sus sentimientos son de luto, que está poseída por la pena de la pérdida de su padre. Sin embargo, aun cuando ese sentimiento está presente en su lamento, Konstan nos hace correctamente notar que el núcleo de lo que ella siente no es pena, sino ira. No es únicamente la muerte del padre lo que deplora, sino la manera en que fue asesinado, y por quiénes fue asesinado. Si Agamenón hubiese muerto en combate, en Troya, Electra se hubiera lamentado también, pero hubiese sido una lamentación distinta, una lamentación sin ira, y no como la que oímos al inicio de la obra (cfr. Konstan, 2001, p. 249).

Más aún, la pena por la pérdida no es el sentimiento en el centro de la obra, ni la emoción con la que se defina a Electra y con la que el público empatizará. La obra no gira en torno al consuelo de la heroína, como hubiese tenido que ser si se la hubiese caracterizado principalmente con la actitud del luto, sino que se estructura más bien a la idea de la venganza, de la reparación debida por un mal causado, idea que de hecho es ajena a la aflicción por la pérdida de un ser querido:

Grief, however, invites no compensatory act, attempt to redress a wrong or a disequilibrium in one's social world. There is no agent who bears responsibility for one's grief, no one to get even with. Of course, loss can be consequence of an action, as when someone, through deliberate aggression or culpable negligence, brings about the death of another (along with damage to one's public self or reputation). In that case, however, we hate or are angry with the author of the deed, but the sense of bereavement has as its object not the one who caused the loss but the loved one who passed away (Konstan, 2001, p. 248).

El lamento con el que se introduce a Electra hace inmediatamente hincapié en el agravio al padre, que es también penoso para la hija. En efecto, el criminal asesinato ha hecho odiosos los lechos de la casa de Agamenón. De hecho, su lamento acaba elocuentemente con la invocación a la venganza, luego de poner por testigos del crimen a los dioses infernales: 
Erinias, ilustres hijas de los dioses, que contempláis a los que han muerto injustamente, a los que han sido engañados en sus lechos, venid, socorredme, vengad el asesinato de mi padre y haced venir a mi hermano, pues sola no soy capaz de llevar equilibrado el peso de la pena que cargo al otro lado (vv. 111-121).

El primer lamento de Electra (vv. 88-121) es clave, pues, además de que por él conocemos por primera vez a la protagonista, y de que resume el problema, y los sentimientos, centrales de la obra, también tiene la virtud de plantear claramente la separación de amigos y enemigos: Electra y Orestes, que son además los ofendidos, por un lado; y Egisto y Clitemestra, los ofensores, por el otro. Ambos bandos, además, necesitan estar unidos para prevalecer sobre el otro: así, Electra se mueve con libertad cuando Egisto no está (vv. 518-519), mientras que no es capaz de llevar a cabo la venganza en ausencia de Orestes (v.120).

La existencia de una ofensa, de un desprecio, es la condición basal para que pueda hablarse entre los griegos de enojo. Esta emoción está acompañada por el dolor que deja la ofensa, pero también por el placer: "anger is accompanied not just by pain but also by pleasure, too, which derives from the desire to avenge the slight that has been suffered: for this desire is accompanied by the expectation (elpis) of its fulfillment, which we regard as possible" (Konstan, 2001, p. 42). No es difícil ver la coincidencia entre esta descripción y la actitud de Electra, que se lamenta y alimenta su ira, pero no desespera, pues confía en la llegada del vengador. Sólo oiremos en su boca la desesperación, y por breve espacio de tiempo, cuando crea que Orestes ha muerto (v. 674 y, especialmente vv. 824 y ss.), y solo hasta que se convenza a sí misma de tomar la venganza por su propia mano.

Sin embargo, creemos que los sentimientos de Electra no son principalmente de ira, sino más bien de odio (aunque haya igualmente ira en ella), por dos razones:

Primero, la naturaleza del enojo, como era entendida entre los griegos, implicaba una valoración de los roles y posición social del ofensor y del ofendido. No cualquiera puede despreciar injustamente a otro (esta es la causa de la ira), sino solo aquel que debió haber respetado al ofendido y que, pese a ello, le despreció (cfr. Konstan, 2001, pp. 45-46). Aunque esto calza en parte con la situación de Electra, hace al mismo tiempo la ira incompatible con el miedo: "Aristotle says that 'it is imposible to be afraid 
of and angry with someone at the same time' $(2.3,1380 \mathrm{a} 33)$. The reason for this, I presume, is that acknowledge, by our fear, the superior status of the other, who is accordingly in a fit position to deliver a slight" (Konstan, 2001, pp. 46-47).

Ahora bien: Electra teme a Egisto, y por eso se siente con mayor libertad de palabra cuando él no está cerca (vv. 312-313). Situación ésta conocida tanto por el coro con el que dialoga (vv. 314-315) como por la misma Clitemestra, que se lo saca en cara (vv. 517-520). No puede, en consecuencia, sentir enojo en contra de Egisto, pese a su cólera, pero puede en cambio sentir odio, y lo siente en efecto, según veremos.

Precisamente, el segundo motivo por el que lo que guía a Electra no es la ira entendida en el modo en que los griegos hacían, es que el enojo tiene por finalidad el sufrimiento del ofensor, un sufrimiento del que él tiene que ser tan consciente como el ofendido lo fue de la ofensa: solo así se cumple la venganza. La muerte del ofensor no es deseada por el ofendido:

That is why we may wish that people whom we hate should die, but when we are angry, what we desire is that the other person feel in return (antipathein) the kind of diminishment that provoked our anger on the first place ... the death of the other would render that impossible (Konstan, 2001, p. 47).

Es claro que el deseo más vehemente de Electra es la muerte de Egisto y Clitemestra. Es este un sentimiento muy duro y fuerte, que avanza en ella incluso por sobre la vergüenza confesada que le produce tener que actuar en contra del natural decoro que debiese guardar una hija respecto de su madre. Sin embargo, es lo que corresponde desde el momento en que ella y Egisto se han transformado en enemigos y la tratan como tal: desear la muerte de ambos es propio del odio.

El odio para con el enemigo era concebido como una emoción moral (Konstan, 2001, p. 200), pues implica una valoración de la persona odiada como viciosa, (Konstan, 2001, p. 190). Por ende, no solo sirve como móvil para la venganza, sino como justificación de la misma. Y al mismo tiempo, y quizá sea lo más importante, implica una caracterización del enemigo, de modo que quien empatice con los sentimientos de Electra, deberá también reconocer la maldad de Clitemestra y Egisto, y aplaudir por tanto sus muertes como lo que tenían merecido. 
In Sophocles' Electra, the heroine recalls how her mother abused her, calling her a 'hateful creature [misêma] loathesome to the gods' (289). In turn, Electra accuses her sister Chrysostemis of cowardice for failing to make manifest in action her hatred (misos) for her mother (347-8), and reproaches her (357-8): 'for me, you are a hater who hates in words, but in fact you live with our fathers assassins' (Konstan, 2001, pp. 187-188).

Esta es, entonces, la emoción que gobierna los actos de la apasionada Electra. Mas es un odio dirigido hacia un fin: Electra busca venganza, una venganza que a sus ojos es también justicia. En esto, no es distinta de los demás héroes: el orden se ha roto al inicio de la obra, y ese orden debe ser restablecido. A medida que la obra avanza, toda la acción, que en la práctica son los parlamentos de Electra, se centra en las líneas que se van dibujando para separar a amigos de enemigos.

Sófocles vuelve a demostrar aquí su interés en centrar su tragedia en el personaje de Electra por sobre cualquier acción dramática al ir caracterizando a los personajes que entran en escena casi exclusivamente según sus relaciones con la protagonista, y no tanto según su posición respecto del crimen de Agamenón, que es lo que inicialmente creó los dos grupos de philoi (Whitlock Blundell, 1989, p. 151). Los diálogos, en cambio, no se centran para caracterizar a los personajes en este hecho, sino en si demuestran interés o desinterés para con la sufriente Electra, compasión o desprecio por su situación. Veamos algunos casos:

Crisótemis se acerca a Electra como hermana, pese a haber adoptado una postura muy distinta a la suya respecto del crimen de su madre. Sin embargo, no es un personaje que nos resulte odioso, o que en la obra se perciba como enemigo, pese a recibir alguna invectiva de Electra respecto de lo que ésta considera cobardía. No. Crisótemis puede ser la antítesis de lo que Electra representa como decisión ante la injusticia, pero lo que la mueve a dialogar con su hermana es cierta piedad fraterna: Crisótemis no quiere ver sufrir a Electra ni que ésta siga acumulando desgracias sobre su vida, así lo declara al coro, cuando explica por qué ha decidido venir a advertirle: 
Yo, mujeres, de alguna manera estoy acostumbrada a las razones de ésta, y no le hubiera dicho nada, si no hubiera oído que una tremenda desgracia se abate sobre ella, tal que la contendrá en sus largos lamentos (vv. 372-376).

Es el deseo de evitarle una nueva desgracia lo que mueve a Crisótemis a hablar con su hermana, así como será el deseo de darle una alegría el que la moverá a correr hasta ella para comunicarle la llegada de Orestes (vv. 871 y ss.) y el que la encontrará dispuesta en ayudar en lo que sea necesario para ejecutar la venganza (v. 944), aunque cuando se entere de que ésta pretende realizarla por propia mano tratará de disuadirla de una locura que, de nuevo, amenaza con traerle mayores desgracias que beneficios (vv. 993 y ss.). Crisótemis, aunque finalmente dejará la escena distanciada de su hermana por la disparidad de sus visiones, es sin embargo el personaje que está siempre preocupado por ella (v. 1036). Estos sentimientos de philia hacia la protagonista llevarían al público a valorarla positivamente, así como a ponderar sus argumentos como razonables, pero, y esto es importante, no como heroicos. La postura de Crisóstemis es entendible, pero no es ejemplar.

Similares sentimientos para con Electra definen también al coro de mujeres argivas. Éstas también desean lo mejor para la protagonista, por lo que la aconsejan y la consuelan, pensando en principio que sus lamentos son solo debidos al duelo. Como afirma Whitlock, el coro toma respecto de Electra el rol de madre (1989, p. 152). Ahora bien, el coro, a diferencia de Crisótemis, va manifestando una admiración creciente por Electra y su temple, y es el único que alabará su valentía de proponerse realizar la venganza por su propia mano, cuando crea que Orestes ha muerto (vv. 1070 y ss.). Junto a Electra, el coro se alegra de la muerte de los tiranos (vv. 1508-1510) y se convence absolutamente de la causa de Electra y de Orestes. Hasta cierto punto, podríamos afirmar que las emociones del coro irían coincidiendo con las del público, de modo que, como éste, los espectadores terminarían por alabar a Electra por su valentía (cosa que no deja de resultar paradójica, si consideramos que no realiza finalmente ninguna acción física) y por desear y alegrarse con el fin de sus enemigos.

Tenemos también a Orestes, cuyas relaciones con Electra están también marcadas por la compasión. Si en un primer momento trata de mantenerse frío y en oculto para poder realizar sus planes, no podrá menos que, viendo las desgracias que padece su hermana, sentir al menos piedad por 
ella (vv. 1198-1199). Esto es lo que le lleva a revelarle su identidad (vv. 1222-1224), arriesgando con ello su misión, pues las muestras de alegría de ésta bien pudieron alertar a todos en el palacio, de no haber estado dentro el pedagogo, como él mismo les recrimina al aparecer en escena (vv. 1326 y ss.). Una vez más, si la obra estuviera centrada en la acción dramática de la venganza de Orestes, este diálogo y situación sería del todo superflua. Pero si, como afirmamos, la obra tiene por objetivo realzar el ethos de Electra, entonces la escena del reconocimiento sirve tanto para mostrar una faceta nueva de la protagonista (su desbordante alegría), como para caracterizar a Orestes como una figura amiga, capaz de sentir compasión por su hermana.

Y quizá lo más importante, luego de la fugaz alegría del reconocimiento, cuando el público quisiera desbordarse al ver por fin que la fortuna comienza a sonreír a la protagonista, es que debe ésta reprimir su expansión, dando un tinte amargo a la escena, para evitar que una excesiva alegría eche por el suelo la realización de la justicia final: de nuevo la obra caracteriza a Electra como un personaje sufriente, al que pareciera le está vedado todo gozo. Elocuente al respecto, por lo gráfica, es la antístrofa entre los dos hermanos, en que ella canta sus versos mientras él, más consciente del peligro, solo los recita, aunque llega a cantarlos luego, en el epodo antes de la entrada del pedagogo (vv. 1234-1287).

Como vemos, los amigos de Electra son caracterizados según las emociones que su personalidad única les suscita. Pero esto no ocurre solo entre los amigos: idéntica cosa sucede entre sus enemigos. Veamos el caso más importante, el de Clitemestra, a quien se la representa en una larga discusión con su hija.

Quizá lo que más hace despreciable a ojos del público a este personaje es precisamente el sentimiento que representa: Clitemestra odia a su hija: así como se alegra de la muerte de Orestes, desearía igual suerte para Electra (v. 791). Como sabemos, desear la muerte es propio del odio. Trata Clitemestra de justificarse ante la misma Electra, atribuyendo a ésta características viciosas (vv. 516-552), que luego resume la misma Electra: "proclama ante todos, si quieres, que soy malvada y deslenguada y llena de desvergüenza" (vv. 606-607), es decir, atributos que la harían odiable y que por ende avalarían la actitud de Clitemestra respecto de ella (y la transformarían, por lo tanto, en la verdadera heroína trágica). Sin embargo, la obra está centrada en Electra y la emocionalidad está con ella: "si por naturaleza soy experta en todas estas cosas" remata Electra apuntando a su madre "tal vez sea porque no desdigo de tu estirpe" (vv. 608-609). Efecti- 
vamente, ambos personajes se odian mutuamente, pero como el odio solo puede legitimar a una de las dos (solo una a la vez puede estar orientando éticamente su odio, por necesidad la otra lo orienta negativamente) esto hace que los intentos de Clitemestra por negativizar a Electra la hagan aún más aborrecible para el público, lo que tiene el efecto adicional de desechar también sus argumentos como inválidos.

Electra desarma la argumentación de su madre, que se amparaba en la justicia de su proceder para con Agamenón por el sacrificio de Ifigenia, sugiriendo que ésta no actúa en realidad por justicia, sino que movida por su impío amor a Egisto, con quien vive ahora (vv. 585-594). Frente a las acusaciones de su hija, la madre no logra mantener la frialdad y deja escapar su enojo ante su desprecio (vv. 626- 629). Odio, ira, desprecio, son los sentimientos que Clitemestra reserva para Electra, y son a su vez los sentimientos que la caracterizan en general en la obra, y por los cuales será finalmente ejecutada.

\section{Electra y el juicio de la tragedia}

Al comienzo de este trabajo revisábamos dos interpretaciones que tratan de dar un sentido a la obra entera: Seaford, por su lado, se centra en lo que llama la destrucción de los límites del luto, mientras que Whitlock, por el suyo, resalta la aplicación de la ley del Talión. Apuntábamos también que ambas interpretaciones son compatibles en la Electra de Sófocles. Sin embargo, en ambos casos la atención se ha puesto en la acción misma para interpretar la obra. Al centrarnos, en cambio, en la emocionalidad, que sería probablemente lo que en primer lugar "golpearía" al público y lo que luego éste retendría, hemos dado realce a la centralidad de la figura de Electra.

Para ella, la destrucción de los límites del luto no es otra cosa que el contexto en que se ve obligada a moverse, las circunstancias fácticas, provocadas por sus enemigos, que la han relegado a un estado deplorable que clama venganza. Y la ley del Talión es, a su vez, el marco en el que ella proyecta sus acciones, el móvil que da razón a sus sentimientos y bajo el que ha estado actuando al ejercer el lamento como una forma de vendetta.

Pero la obra no se trata ni del luto, ni de la venganza. Se trata de Electra, una figura admirable por su ánimo indoblegable, y conmovedora, por sus desgracias. Esta Electra y sus lamentos, que dan vida a gran parte de la obra, tienen una función acusadora: 
Like a Fury, she tortures Clytemnestra, making her fearful and defensive. Her verbal control of the stage increases throughout the first half of the play; Clytemnestra is afraid, for example, to pray openly to Apollo. Electra's stance can only result in completed revenge when the male avenger responds to her words; but in the meantime, she persuades the chorus to join at least partially in her lament, she stirs the timid Chrysothemis to undertake an important religious mission, and she successfully puts her mother "on trial" for her crime (Foley, 2001, p. 151).

Electra es como una Erinis, que clama venganza por la sangre derramada. Sus lamentos y su odio hacia los enemigos de la casa de su padre caracterizan a todos los que están en la escena, acusando a los enemigos y realzando a los amigos. Le mueve una ira apasionada, tal y como a las Erinias de Esquilo, una ira que sobrepuja a su pena por la pérdida de su padre y que la encamina hacia el odio contra su madre.

Electra's wrath sets her actively in conflict with her mother and Aegisthus; it is not merely the psychic residue of a past event but has implications for the present and the future. It is thus a factor in the opposition of wills on which drama depends. To put it differently, the cause of Electra's passion is not just an incident -the passing away of her father - but an action, instigated by agents whom she holds responsible. This social dimension is what distinguishes Electra's emotional response of anger from the passive grief elicited by a morally neutral event. The opening scene of Sophocles' Electra can be read as a subtle examination and confrontation of two distinct kinds of sentiment (Konstan, 2001, p. 251).

Su pasionalidad, tal y como recomienda Gorgias a los oradores, va en apoyo de sus argumentos: la obra de Sófocles no ha sido otra cosa que dar un rostro humano a una de esas palabras poderosas de las que habla Gorgias en su Encomio de Helena (2011, párr. 8). Una palabra que se levanta clamando para que se realice la justicia, apelando al jurado de Atenas, que es el público, tal y como las Erinias de Esquilo acusaron a Orestes ante los ciudadanos en Coéforas. Esta vez, el público-jurado está del lado de esta particular Erinis y Orestes ejecuta la sentencia. 


\section{REFERENCIAS BIBLIOGRÁFICAS}

FOLEY, H. P. (2001). Female Acts in Greek Tragedy. Princeton: Princeton University Press.

GOLDHILL, S. (1986). Text and Tradition. En Reading Greek Tragedy. Cambridge: Cambridge University Press.

GORGIAS. (2011). Encomio de Helena (M. C. Davolio \& G. E. Marcos, Trads.). Buenos Aires: Ediciones Winograd.

GOULD, J. (1983). Homeric Epic and the Tragic Moment. En T. Winnifrith, P. Murray, \& K. W. Gransden, Aspect of the Epic. New York: St. Martin's Press.

KONSTAN, D. (2001). The Emotions of the Ancient Greeks, Studies in Aristotle and Classical Literature. Toronto; Buffalo; London: University of Toronto Press.

ROSENWEIN, B. H. (2003). Even the Devil (Sometimes) has Feelings: Emotional Communities in the Early Middle Ages. Haskins Society journal, 14, 1-14.

--- (2006). Emotional communities in the early middle ages (1st ed.). Ithaca: Cornell University Press.

SEAFORD, R. (1985). The Destruction of Limits in Sophokes' Elektra. The Classical Quarterly, 35(2), 315-323.

SÓfOCLES. (2000). Electra. En A. Alamillo (Trad.), Tragedias (De Sófocles). Madrid: Gredos.

WHITLOCK BLUNDELL, M. (1989). Helping Friends and Harming Enemies, a study in Sophocles and Greek Ethics. Cambridge: Cambridge University Press. 